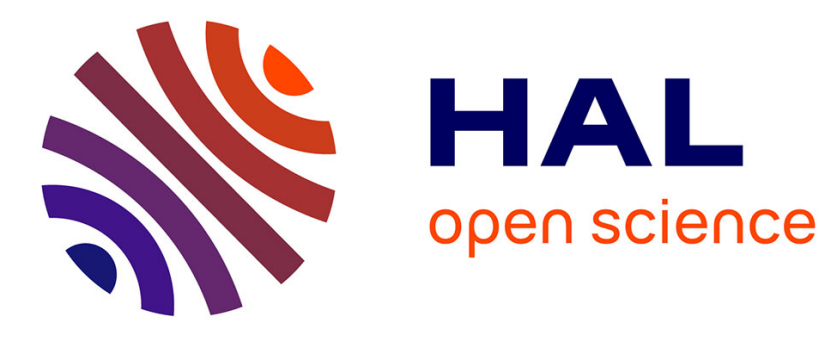

\title{
Design of Interval Observers for Uncertain Linear Impulsive Systems
}

Kwassi H Degue, Denis Efimov, Jerome Le Ny, Sandra Hirche

\section{To cite this version:}

Kwassi H Degue, Denis Efimov, Jerome Le Ny, Sandra Hirche. Design of Interval Observers for Uncertain Linear Impulsive Systems. Proc. 60th IEEE Conference on Decision and Control (CDC), Dec 2021, Austin, United States. hal-03439137

\section{HAL Id: hal-03439137 https://hal.inria.fr/hal-03439137}

Submitted on 22 Nov 2021

HAL is a multi-disciplinary open access archive for the deposit and dissemination of scientific research documents, whether they are published or not. The documents may come from teaching and research institutions in France or abroad, or from public or private research centers.
L'archive ouverte pluridisciplinaire HAL, est destinée au dépôt et à la diffusion de documents scientifiques de niveau recherche, publiés ou non, émanant des établissements d'enseignement et de recherche français ou étrangers, des laboratoires publics ou privés. 


\title{
Design of Interval Observers for Uncertain Linear Impulsive Systems
}

\author{
Kwassi H. Degue, Denis Efimov, Jerome Le Ny and Sandra Hirche
}

\begin{abstract}
This paper presents a novel approach to design interval estimators for uncertain linear impulsive systems. We consider situations where model disturbances and measurement noises are unknown but lie in given intervals. We propose a new architecture providing more degrees of freedom than standard interval observer structures for linear impulsive systems. We test the efficiency of the proposed methodology through numerical simulations.
\end{abstract}

\section{INTRODUCTION}

State estimation is critically important in numerous control applications, as measurements of all state variables are often costly and difficult to obtain. Accordingly, multifarious approaches have been proposed to design state observers [1]. Frequently, these methodologies focus on the design of point-wise observers, e.g., Luenberger observers, which provide estimates converging to the state's true values, when possible. Stochastic observers such as Kalman filters also provide a confidence measure around the point estimates, but require that the statistical properties of the systems' disturbances are known [2]. However, for industrial systems, the state disturbances and measurement noises can often be modeled as bounded signals, whereas their statistical properties are harder to estimate. Interval estimators [3], [4] are then advantageous, as they can handle the presence of disturbances whose values are only known to belong to given intervals or polytopes, and many types of bounded uncertainties more generally.

The NASA University Leadership Initiative (grant \#80NSSC20M0163) provided funds to assist $\mathrm{K}$. H. Degue with his research, but this article solely reflects the opinions and conclusions of its authors and not any NASA entity. This work was supported in part by NSERC under Grant RGPIN-5287-2018 and RGPAS-2018-522686, the Ministry of Science and Higher Education of Russian Federation (passport of goszadanie no. 20190898), and the European Research Council (ERC) Consolidator Grant "Safe data- driven control for human-centric systems (COMAN)" under grant agreement number 864686 .

K. H. Degue is with the Department of Aeronautics and Astronautics at the Massachusetts Institute of Technology, Cambridge, MA. kdegueamit.edu

J. Le Ny is with the department of Electrical Engineering, Polytechnique Montreal and GERAD, QC H3T-1J4, Montreal, Canada. jerome.le-ny@polymtl.ca

D. Efimov is with Inria, Univ. Lille, CNRS, UMR 9189 - CRIStAL, F-59000 Lille, France and ITMO University, 197101 Saint Petersburg, Russia. Denis.Efimoveinria.fr

S. Hirche is with the Chair of Information-oriented Control, Technical University of Munich, 80333, Germany. hirchedlsr.ei.tum.de
Impulsive systems form an important class of hybrid systems that includes both discrete and continuous event dynamics. Interval observers have been proposed for linear impulsive systems in [5]-[7]. The approach in [7] requires the estimation error dynamics to be stable and cooperative, which may be restrictive since it may not be possible to determine an observer gain that ensures these properties. To address this issue, coordinate transformations are commonly used, when the system is observable [5], [6]. Although such methods achieve reasonable accuracy, a drawback of interval observer design based on coordinate transformation is that such estimators can provide conservative bounds since the transformations have a strong impact on performance. Another drawback of such methods is that there is no constructive approach to compute the coordinate transformations and the interval observer gain simultaneously, in order to satisfy the cooperative property and disturbance rejection constraints [8]. Among recently proposed alternative methods, [9] presents a two-step approach that integrates reachability analysis and robust observer design, and [8] includes an additional degree of freedom into the design of an interval observer. However, these two approaches have been proposed only for discretetime linear systems, while impulsive systems, which can be used to model a wide variety of real-world processes, e.g., systems with impacts [10], have not been considered.

The contribution of this paper consists in designing a new interval observer for uncertain linear impulsive systems. Motivated by [8], we develop a novel observer structure whose design conditions are less conservative than those of standard interval observers, since it provides two additional degrees of freedom. These degrees of freedom improve the accuracy of interval estimation. The structure of our interval observer circumvents the issue of coordinate transformation and provides more accurate estimates than interval observers based on such transformations. Furthermore, we prove the input-to-state stability (ISS) property of linear impulsive systems under minimum dwell-time constraints ${ }^{1}$ when some linear matrix inequalities (LMIs) are satisfied.

Section II presents the problem statement. Section III recalls some well-known nonnegativity conditions for continuous-time and discrete-time systems and some basic

\footnotetext{
${ }^{1}$ Dwell-times are times between two consecutive impulses.
} 
facts about interval observer design as well as the stability analysis of impulsive systems under minimum dwell-time constraints. The stability conditions are used in Section III to design the novel interval observer. Finally, we illustrate the performance of the methodology through numerical simulations in Section IV.

Notation. We denote the real numbers by $\mathbb{R}$, the integers by $\mathbb{Z}, \mathbb{R}_{\geq 0}=\{\tau \in \mathbb{R}: \tau \geq 0\}$ and $\mathbb{Z}_{\geq 0}=\mathbb{Z} \cap \mathbb{R}_{\geq 0}$. We denote the cones of vectors of dimension $n$ with positive and nonnegative components by $\mathbb{R}_{>0}^{n}$ and $\mathbb{R}_{\geq 0}^{n}$ respectively. We denote the $p$-norm of a vector $x \in \mathbb{R}^{n}$ by $|x|_{p}:=\left(\sum_{i=1}^{k}\left|x_{i}\right|^{p}\right)^{1 / p}$, for $p \in[1, \infty)$, and $|x|_{\infty}:=$ $\max _{i \in\{1, \ldots, n\}}\left|x_{i}\right|$. For a measurable vector-valued signal $u: \mathbb{R}_{\geq 0} \rightarrow \mathbb{R}^{n}$, we denote its $L_{\infty}$-norm as $\|u\|_{L_{\infty}}:=$ $\operatorname{ess~sup}_{t>0}|u(t)|_{2}$. We denote by $\mathcal{L}_{\infty}^{n}$ the set of signals $u$ with the property $\|u\|_{L_{\infty}}<\infty$. The $n \times n$ identity matrix is denoted $I_{n}$. For two vectors $x_{1}, x_{2} \in \mathbb{R}^{n}$ or matrices $A_{1}, A_{2} \in \mathbb{R}^{n \times n}$, the relations $x_{1} \leq x_{2}$ and $A_{1} \leq A_{2}$ are understood element-wise. A matrix $A \in \mathbb{R}^{n \times n}$ is Hurwitz if all its eigenvalues have negative real parts; it is called Schur stable if all its eigenvalues have absolute value less than one; it is called Metzler if all its elements outside of the main diagonal are nonnegative and it is called nonnegative if $A \geq 0$. The notation $P \succ 0$ ( $P \succeq 0$ ) for a symmetric matrix $P \in \mathbb{R}^{n \times n}$ means that it is positive (nonnegative) definite. We denote the set of $n \times n$ positive definite matrices by $S_{\succ 0}^{n}$. In a symmetric block matrix, the symbol $*$ denotes a term that can be deduced by symmetry.

\section{Problem Statement}

Consider the following continuous-time hybrid (impulsive) linear system

$$
\begin{aligned}
\dot{x}(t) & =A x(t)+w(t), \quad \forall t \in\left[t_{i}, t_{i+1}\right), i \in \mathbb{Z}_{\geq 0}, \\
x\left(t_{i+1}\right) & =G x\left(t_{i+1}^{-}\right)+g\left(t_{i+1}\right), \quad \forall i \in \mathbb{Z}_{\geq 0}, \\
y(t) & =C x(t)+v(t), \quad \forall t \geq t_{0},
\end{aligned}
$$

where $x(t) \in \mathbb{R}^{n}$ represents the state vector and $x\left(t_{i+1}^{-}\right)$ stands for the left-sided limit of $x(t)$ for $t \rightarrow t_{i+1} ; A, G \in$ $\mathbb{R}^{n \times n} ; w \in \mathcal{L}_{\infty}^{n}$ represents the input for $t \in\left[t_{i}, t_{i+1}\right)$; a continuous signal $g \in \mathcal{L}_{\infty}^{n}$ stands for the input at time instants $t_{i+1}$ for $i \geq 1 ; y(t) \in \mathbb{R}^{p}$ represents the output signal available for measurements; $v \in \mathcal{L}_{\infty}^{p}$ represents the measurement noise; $C \in \mathbb{R}^{p \times n}$. We assume that the sequence of impulse events $t_{i}$ with $i \in \mathbb{Z}_{\geq 0}$ is positively incremental, i.e., $\theta_{i}=t_{i+1}-t_{i}>0$ and $t_{0}=0$. In addition, we assume that the matrices $A, C$ and $G$ are given. We do not impose any stability condition on the matrices $A$ and $G$. We assume that the system (1) evolves under predetermined mode transitions, i.e., the impulse instants $t_{i}$ are given, and moreover $\theta_{i} \in\left[T_{\min },+\infty\right)$ for all $i \in \mathbb{Z}_{\geq 0}$, where $T_{\min }$ is a given positive constant.
Throughout this paper, we assume that initial conditions for $x(0)$, instantaneous values of time-varying inputs $w$ and $g$ in the continuous and discrete dynamics, and instantaneous values of the measurement noise $v$ are uncertain but belong to given intervals. Namely, four bounds $\underline{v}, \bar{v} \in \mathbb{R}^{p}$ and $\underline{x}(0), \bar{x}(0) \in \mathbb{R}^{n}$ are given such that

$$
\underline{v} \leq v(t) \leq \bar{v}, \forall t \geq 0 \text {, and } \underline{x}(0) \leq x(0) \leq \bar{x}(0) .
$$

Furthermore, two functions $\underline{w}, \bar{w} \in \mathcal{L}_{\infty}^{n}$ are given such that

$$
\underline{w}(t) \leq w(t) \leq \bar{w}(t), \quad \forall t \in \mathbb{R}_{\geq 0},
$$

and two functions $\underline{g}, \bar{g} \in \mathcal{L}_{\infty}^{n}$ are given such that

$$
\underline{g}(t) \leq g(t) \leq \bar{g}(t), \quad \forall t \in \mathbb{R}_{\geq 0} .
$$

The goal of this paper is to design an interval observer, i.e., we want compute at each time $t \geq 0$ lower and upper bounds for the state $x(t)$ such that $\underline{x}(t) \leq x(t) \leq$ $\bar{x}(t)$. In particular, we aim to circumvent the coordinate transformation issue of [5], [6] by designing an interval observer with more degrees of freedom.

\section{INTERVAL OBSERVER DESIGN}

In this section, we design an interval observer for the system (1). First, we review some basic facts from interval estimation, impulsive systems and positive linear systems, which are needed in the sequel.

\section{A. Preliminaries on interval analysis}

Given a matrix $A \in \mathbb{R}^{m \times n}$, define $A^{+}=\max \{0, A\}$, $A^{-}=A^{+}-A$ (we use the same notation for vectors) and denote the matrix of absolute values of all elements by $|A|=A^{+}+A^{-}$.

Lemma 1. [11] Let $x \in \mathbb{R}^{n}$ be a vector variable with $\underline{x} \leq x \leq \bar{x}$ for some given vectors $\underline{x}, \bar{x} \in \mathbb{R}^{n}$. If $A \in \mathbb{R}^{m \times n}$ is a constant matrix, then

$$
A^{+} \underline{x}-A^{-} \bar{x} \leq A x \leq A^{+} \bar{x}-A^{-} \underline{x} .
$$

\section{B. Nonnegative linear systems}

1) Nonnegative continuous-time linear systems: Next, consider the following LTI system

$$
\begin{aligned}
\dot{x}(t) & =A x(t)+B \omega(t), \omega: \mathbb{R}_{\geq 0} \rightarrow \mathbb{R}_{\geq 0}^{q}, \forall t \in \mathbb{R}_{\geq 0} \\
y(t) & =C x(t)+D \omega(t),
\end{aligned}
$$

with $x \in \mathbb{R}^{n}, y \in \mathbb{R}^{p}$ and $A \in \mathbb{R}^{n \times n}$ is a Metzler matrix.

Lemma 2. [12] If $x(0) \geq 0$ and $B \in \mathbb{R}_{>0}^{n \times q}$, any solution of the LTI system (4) is element-wise nonnegative. Furthermore, the output solution $y(t)$ is nonnegative if $C \in \mathbb{R}_{\geq 0}^{p \times n}$ and $D \in \mathbb{R}_{\geq 0}^{p \times q}$. A dynamical system that satisfies all these restrictions is called cooperative. 
2) Nonnegative discrete-time linear systems: Consider the system

$$
x_{t+1}=A x_{t}+B \omega_{t}, \omega: \mathbb{Z}_{\geq 0} \rightarrow \mathbb{R}_{\geq 0}^{m}, t \in \mathbb{Z}_{\geq 0}
$$

with $x_{t} \in \mathbb{R}^{n}, A \in \mathbb{R}_{\geq 0}^{n \times n}$ and $B \in \mathbb{R}_{\geq 0}^{n \times m}$.

Lemma 3. [12] If $x(0) \geq 0$, then any solution of (5) is element-wise nonnegative for all $t \in \mathbb{Z}_{\geq 0}$.

C. Stability of linear impulsive systems under minimum dwell-time constraints

The next theorem recalls a result about stability of linear impulsive systems under minimum dwell-time constraints.

Theorem 1. [13, Lemma 2.2] Consider the system (1) with $w \equiv 0, g \equiv 0$ and dwell-times $\theta_{i} \in\left[T_{\min },+\infty\right)$ for all $i \in \mathbb{Z}_{\geq 0}$, where $T_{\min }$ is a given constant. If there exists a matrix $P \in S_{\succ 0}^{n}$ such that

$$
\begin{array}{r}
A^{T} P+P A \prec 0, \\
G^{T} e^{A^{T} T_{\min }} P e^{A T_{\min }} G-P \prec 0,
\end{array}
$$

then the system (1) is asymptotically stable.

In the context of interval estimation, in addition to the global asymptotic stability (GAS) property, one needs to prove the input-to-state stability (ISS) property (see the definition of ISS in [14]) of the estimation errors' dynamics. This motivates the results of the following section.

D. Robust stability of linear impulsive systems under minimum dwell-time

Next, we prove a new result to guarantee the ISS property with respect to the inputs $w$ and $g$, under a dwell-time constraint.

Theorem 2. Let all conditions of Theorem 1 be satisfied, $w \in \mathcal{L}_{\infty}^{n}$, and $g \in \mathcal{L}_{\infty}^{n}$ be continuous. Then the system (1) is ISS with respect to the inputs $w$ and $g$.

Proof. Consider for the system (1) a Lyapunov function candidate $V(x)=x^{\mathrm{T}} P x\left(\right.$ denote $\left.V(t)=x(t)^{\mathrm{T}} P x(t)\right)$ with $P \in S_{\succ 0}^{n}$. We have

$$
\begin{aligned}
\dot{V}(t) & =x^{\mathrm{T}}(t)\left(A^{\mathrm{T}} P+P A\right) x(t)+2 x^{\mathrm{T}}(t) P w(t) \\
& =\left[\begin{array}{c}
x(t) \\
w(t)
\end{array}\right]^{\mathrm{T}} Q_{1}\left[\begin{array}{c}
x(t) \\
w(t)
\end{array}\right]-\alpha_{1} V(t)+\gamma_{1}|w(t)|_{2}^{2}, \\
Q_{1} & =\left[\begin{array}{cc}
A^{\mathrm{T}} P+P A+\alpha_{1} P & P \\
P & -\gamma_{1} I_{n}
\end{array}\right], \alpha_{1}, \gamma_{1}>0,
\end{aligned}
$$

for all $t \in\left[t_{i}, t_{i+1}\right)$ and for any $i \in \mathbb{Z}_{\geq 0}$. Moreover, by taking $\alpha_{1}$ small and $\gamma_{1}$ large enough, the following inequality is satisfied

$$
A^{\mathrm{T}} P+P A+\alpha_{1} P+\frac{1}{\gamma_{1}} P P \prec 0,
$$

since the first LMI in (6) is satisfied and $P$ is a symmetric matrix. Consequently, if the strict LMIs in (6) are satisfied, by using a Schur complement, there exist values for $\alpha_{1}$ small and $\gamma_{1}$ such that $Q_{1} \preceq 0$. Then,

$$
\dot{V}(t) \leq-\alpha_{1} V(t)+\gamma_{1}\|w\|_{\infty}^{2}, \forall t \in\left[t_{i}, t_{i+1}\right), i \in \mathbb{Z}_{\geq 0} .
$$

Define

$$
\begin{aligned}
& \theta_{i}=t_{i+1}^{-}-t_{i}, J_{i}=\int_{0}^{\theta_{i}} e^{A \tau} w\left(t_{i+1}^{-}-\tau\right) d \tau, \\
& \Phi_{i}=e^{A \theta_{i}}, \Phi_{\min }=e^{A T_{\min }} .
\end{aligned}
$$

We deduce from (1) that

$$
\begin{aligned}
x\left(t_{i+1}^{-}\right) & =e^{A \theta_{i}} x\left(t_{i}\right)+\int_{t_{i}}^{t_{i+1}^{-}} e^{A\left(t_{i+1}^{-}-s\right)} w(s) d s, \\
& =e^{A \theta_{i}} x\left(t_{i}\right)+\int_{0}^{\theta_{i}} e^{A \tau} w\left(t_{i+1}^{-}-\tau\right) d \tau, \\
& =\Phi_{i}\left(G x\left(t_{i}^{-}\right)+g\left(t_{i}\right)\right)+J_{i}, \\
& =\Phi_{i} G x\left(t_{i}^{-}\right)+\Phi_{i} g\left(t_{i}\right)+J_{i} .
\end{aligned}
$$

Then, we get

$$
\begin{gathered}
V\left(t_{i+1}^{-}\right)=\left(x\left(t_{i}^{-}\right)\right)^{\mathrm{T}} G^{\mathrm{T}} \Phi_{i}^{\mathrm{T}} P \Phi_{i} G x\left(t_{i}^{-}\right)+ \\
2\left(x\left(t_{i}^{-}\right)\right)^{\mathrm{T}} G^{\mathrm{T}} \Phi_{i}^{\mathrm{T}} P\left(\Phi_{i} g\left(t_{i}\right)+J_{i}\right)+ \\
\left(\Phi_{i} g\left(t_{i}\right)+J_{i}\right)^{\mathrm{T}} P\left(\Phi_{i} g\left(t_{i}\right)+J_{i}\right) .
\end{gathered}
$$

If the first LMI in (6) is satisfied, the function $\mathcal{V}(t)=$ $e^{A^{\mathrm{T}} t} P e^{A t}$ is decreasing. So in particular, under minimum dwell-time constraints, i.e., when $\theta_{i} \geq T_{\min }$, we get

$$
\begin{aligned}
& e^{A^{\mathrm{T}} \theta_{i}} P e^{A \theta_{i}} \preceq e^{A^{\mathrm{T}} T_{\min }} P e^{A T_{\text {min }}}, \\
& \Rightarrow G^{\mathrm{T}} e^{A^{\mathrm{T}} \theta_{i}} P e^{A \theta_{i}} G \preceq G^{\mathrm{T}} e^{A^{\mathrm{T}} T_{\min }} P e^{A T_{\min }} G .
\end{aligned}
$$

It can be inferred from (8) and (9) that

$$
\begin{aligned}
V\left(t_{i+1}^{-}\right) & \leq\left(x\left(t_{i}^{-}\right)\right)^{\mathrm{T}}\left(G^{\mathrm{T}} \Phi_{\min }^{\mathrm{T}} P \Phi_{\min } G-\alpha_{2} P\right) x\left(t_{i}^{-}\right)+ \\
& 2\left(x\left(t_{i}^{-}\right)\right)^{\mathrm{T}} G^{\mathrm{T}} \Phi_{i}^{\mathrm{T}} P\left(\Phi_{i} g\left(t_{i}\right)+J_{i}\right)+ \\
& \left(\Phi_{i} g\left(t_{i}\right)+J_{i}\right)^{\mathrm{T}} P\left(\Phi_{i} g\left(t_{i}\right)+J_{i}\right) \\
& +\alpha_{2}\left(x\left(t_{i}^{-}\right)\right)^{\mathrm{T}} P x\left(t_{i}^{-}\right),
\end{aligned}
$$

for any $0 \leq \alpha_{2}<1$, independent of $t_{i}$. When (6) is satisfied, $G^{\mathrm{T}} \Phi_{\min }^{\mathrm{T}} P \Phi_{\min } G-\alpha_{2} P \preceq 0$ for some value of $\alpha_{2}$. Consequently, we get

$$
\begin{aligned}
V\left(t_{i+1}^{-}\right) & \leq \alpha_{2}\left(x\left(t_{i}^{-}\right)\right)^{\mathrm{T}} P x\left(t_{i}^{-}\right)+\varphi\left\|\Phi_{i} g\left(t_{i}\right)+J_{i}\right\|_{2}^{2} \\
& +\left[\begin{array}{c}
x\left(t_{i}^{-}\right) \\
\Phi_{i} g\left(t_{i}\right)+J_{i}
\end{array}\right]^{\mathrm{T}} Q_{2}\left[\begin{array}{c}
x\left(t_{i}^{-}\right) \\
\Phi_{i} g\left(t_{i}\right)+J_{i}
\end{array}\right], \\
Q_{2} & =\left[\begin{array}{cc}
G^{\mathrm{T}} \Phi_{\min }^{\mathrm{T}} P \Phi_{\min } G-\alpha_{2} P & * \\
P \Phi_{i} G & P-\varphi I_{n}
\end{array}\right],
\end{aligned}
$$

for any scalar $\varphi$. If the first LMI in (6) is satisfied, $A$ is stable and so $\Phi_{i}$ is bounded. Therefore, when $\varphi$ is large 
enough, by using a Schur complement of $Q_{2}$, we deduce that $Q_{2} \preceq 0$ for all $i$. Furthermore, if the first LMI in (6) is satisfied, $\left|\int_{0}^{\theta_{i}} e^{A \tau} w\left(t_{i+1}^{-}-\tau\right) d \tau\right|_{2} \leq \rho\|w\|_{\infty}$ for some $\rho>0$ and for all $i$, again because $A$ is stable. Consequently, we get

$$
V\left(t_{i+1}^{-}\right) \leq \alpha_{2} V\left(t_{i}^{-}\right)+\gamma_{2} \rho^{2}\|w\|_{\infty}^{2}+\gamma_{3}\|g\|_{\infty}^{2}, \forall i,
$$

for some positive constants $\gamma_{2}$ and $\gamma_{3}$. The inequalities (7) and (10) imply that the system (1) is ISS [14].

E. Interval observer design under minimum dwell-time constraints

We propose a new interval estimator in the form:

$$
\begin{aligned}
& \underline{\dot{\chi}}(t)=(T A-L C) \underline{\eta}(t)+L y(t)+T^{+} \underline{w}-T^{-} \bar{w}- \\
& L^{+} \bar{v}+L^{-} \underline{v}-((T A-L C) N)^{+} \bar{v} \\
& +((T A-L C) N)^{-} \underline{v}, \forall t \in\left[t_{i}, t_{i+1}\right), \\
& \underline{\chi}(0)=T^{+} \underline{x}(0)-T^{-} \bar{x}(0), \\
& \underline{\eta}(t)=\underline{\chi}(t)+N y(t), \forall t \in\left[t_{i}, t_{i+1}\right), \\
& \underline{x}(t)=\underline{\eta}(t)-N^{+} \bar{v}+N^{-} \underline{v}, \forall t \in\left[t_{i}, t_{i+1}\right), \\
& \underline{\chi}\left(t_{i+1}\right)=(R G-M C) \underline{\eta}\left(t_{i+1}^{-}\right)+M y\left(t_{i+1}\right)+R^{+} \underline{g} \\
& -R^{-} \bar{g}-M^{+} \bar{v}+M^{-} \underline{v}-((R G-M C) J)^{+} \bar{v} \\
& +((R G-M C) J)^{-} \underline{v}, \\
& \underline{\eta}\left(t_{i+1}\right)=\underline{\chi}\left(t_{i+1}\right)+J y\left(t_{i+1}\right), \\
& \underline{x}\left(t_{i+1}\right)=\eta\left(t_{i+1}\right)-J^{+} \bar{v}+J^{-} \underline{v}, \\
& \dot{\bar{\chi}}(t)=(T A-L C) \bar{\eta}(t)+L y(t)+T^{+} \bar{w}-T^{-} \underline{w} \\
& +L^{-} \bar{v}-L^{+} \underline{v}+((T A-L C) N)^{-} \bar{v} \\
& -((T A-L C) N)^{+} \underline{v}, \forall t \in\left[t_{i}, t_{i+1}\right), \\
& \bar{\chi}(0)=T^{+} \bar{x}(0)-T^{-} \underline{x}(0) \text {, } \\
& \bar{\eta}(t)=\bar{\chi}(t)+N y(t), \forall t \in\left[t_{i}, t_{i+1}\right) \text {, } \\
& \bar{x}(t)=\bar{\eta}(t)+N^{-} \bar{v}-N^{+} \underline{v}, \forall t \in\left[t_{i}, t_{i+1}\right), \\
& \bar{\chi}\left(t_{i+1}\right)=(R G-M C) \bar{\eta}\left(t_{i+1}^{-}\right)+M y\left(t_{i+1}\right)+R^{+} \bar{g} \\
& -R^{-} \underline{g}+M^{-} \bar{v}-M^{+} \underline{v}+((R G-M C) J)^{-} \bar{v} \\
& -((R G-M C) J)^{+} \underline{v}, \\
& \bar{\eta}\left(t_{i+1}\right)=\bar{\chi}\left(t_{i+1}\right)+J y\left(t_{i+1}\right), \\
& \bar{x}\left(t_{i+1}\right)=\bar{\eta}\left(t_{i+1}\right)+J^{-} \bar{v}-J^{+} \underline{v},
\end{aligned}
$$

for all $i \in \mathbb{Z}_{\geq 0}$, where $\underline{x} \in \mathbb{R}^{n}$ and $\bar{x} \in \mathbb{R}^{n}$ stand, respectively, for the lower and the upper interval estimates for the state $x$. In (11), we have to design the matrices $T, R \in \mathbb{R}^{n \times n}, N, J \in \mathbb{R}^{n \times p}$ and $L, M \in \mathbb{R}^{n \times p}$. Inspired by the structure of a point-wise observer that is proposed in [15], we select the matrices $T, N, R$ and $J$ such that

$$
\begin{aligned}
T+N C & =I_{n}, \\
\text { and } \quad R+J C & =I_{n} .
\end{aligned}
$$

Denote the estimation errors as follows

$$
\begin{aligned}
& \underline{e}(t)=x(t)-\underline{x}(t), \bar{e}(t)=\bar{x}(t)-x(t), \forall t \in \mathbb{R}_{\geq 0}, \\
& \underline{e}_{\eta}(t)=x(t)-\underline{\eta}(t), \bar{e}_{\eta}(t)=\bar{\eta}(t)-x(t), \forall t \in \mathbb{R}_{\geq 0},
\end{aligned}
$$

and for all $i \in \mathbb{Z}_{\geq 0}$

$$
\begin{aligned}
& \underline{e}_{\chi}(t)=T x(t)-\underline{\chi}(t), \quad \forall t \in\left(t_{i}, t_{i+1}\right), \\
& \bar{e}_{\chi}(t)=\bar{\chi}(t)-T x(t), \quad \forall t \in\left(t_{i}, t_{i+1}\right), \\
& \underline{e}_{\chi}\left(t_{i+1}\right)=R x\left(t_{i+1}\right)-\underline{\chi}\left(t_{i+1}\right), \\
& \bar{e}_{\chi}\left(t_{i+1}\right)=\bar{\chi}\left(t_{i+1}\right)-R x\left(t_{i+1}\right) .
\end{aligned}
$$

We need the following assumptions.

Assumption 1. There exist matrices $L \in \mathbb{R}^{n \times p}, M \in$ $\mathbb{R}^{n \times p}, T \in \mathbb{R}^{n \times n}, R \in \mathbb{R}^{n \times n}$ and $P \in S_{\succ 0}^{n}$ such that

(i) the following LMIs hold

$(T A-L C)^{T} P+P(T A-L C) \prec 0$

$(R G-M C)^{T} e^{(T A-L C)^{T} T_{\min }} P e^{(T A-L C) T_{\min }}(R G-M C)$

$-P \prec 0$

(ii) the matrix $(T A-L C)$ is Metzler;

(iii) the matrix $(R G-M C)$ is nonnegative.

Theorem 3. Let Assumption 1 be satisfied. Then the estimates $\underline{x}(t)$ and $\bar{x}(t)$ given by (11) yield the relations

$$
\underline{x}(t) \leq x(t) \leq \bar{x}(t), \forall t \in \mathbb{R}_{\geq 0},
$$

and we get $\underline{e}, \bar{e} \in \mathcal{L}_{\infty}^{n}$.

Proof. The dynamics of the errors $\underline{e}_{\chi}, \bar{e}_{\chi}$ can be written as follows for all $i \in \mathbb{Z}_{\geq 0}$

$$
\begin{aligned}
& \dot{e}_{\chi}(t)=(T A-L C) \underline{e}_{\eta}(t)+\sum_{j=1}^{j=3} \underline{\alpha}_{j}(t), \forall t \in\left[t_{i}, t_{i+1}\right), \\
& \dot{\bar{e}}_{\chi}(t)=(T A-L C) \bar{e}_{\eta}(t)+\sum_{j=1}^{j=3} \bar{\alpha}_{j}(t), \forall t \in\left[t_{i}, t_{i+1}\right),
\end{aligned}
$$

with

$$
\begin{aligned}
& \underline{\alpha}_{1}(t)=\left(L^{+} \bar{v}-L^{-} \underline{v}\right)-L v(t), \\
& \underline{\alpha}_{2}(t)=T w(t)-\left(T^{+} \underline{w}-T^{-} \bar{w}\right), \\
& \underline{\alpha}_{3}(t)=((T A-L C) N)^{+} \bar{v}-((T A-L C) N)^{-} \underline{v}, \\
& \bar{\alpha}_{1}(t)=L v(t)-\left(L^{+} \underline{v}-L^{-} \bar{v}\right), \\
& \bar{\alpha}_{2}(t)=\left(T^{+} \bar{w}-T^{-} \underline{w}\right)-T w(t), \\
& \bar{\alpha}_{3}(t)=-\left(((T A-L C) N)^{+} \underline{v}-((T A-L C) N)^{-} \bar{v}\right) .
\end{aligned}
$$


Furthermore, we get

$$
\begin{aligned}
\underline{e}_{\eta}(t) & =x(t)-\underline{\chi}(t)-N C x(t)-N v(t), \forall t \in\left[t_{i}, t_{i+1}\right), \\
& =\underline{e}_{\chi}(t)-N v(t), \forall t \in\left[t_{i}, t_{i+1}\right), \\
\bar{e}_{\eta}(t) & =\bar{\chi}(t)-x(t)+N C x(t)+N v(t), \forall t \in\left[t_{i}, t_{i+1}\right), \\
& =\bar{e}_{\chi}(t)+N v(t), \forall t \in\left[t_{i}, t_{i+1}\right) .
\end{aligned}
$$

Consequently, the errors' dynamics (16) can be rewritten as follows $\forall i \in \mathbb{Z}_{\geq 0}$

$$
\begin{aligned}
& \dot{e}_{\chi}(t)=(T A-L C) \underline{e}_{\chi}(t)+\sum_{j=1}^{j=3} \underline{\beta}_{j}(t), \forall t \in\left[t_{i}, t_{i+1}\right), \\
& \dot{\bar{e}}_{\chi}(t)=(T A-L C) \bar{e}_{\chi}(t)+\sum_{j=1}^{j=3} \bar{\beta}_{j}(t), \forall t \in\left[t_{i}, t_{i+1}\right),
\end{aligned}
$$

with

$$
\begin{aligned}
& \underline{\beta}_{1}(t)=\underline{\alpha}_{1}(t), \quad \underline{\beta}_{2}(t)=\underline{\alpha}_{2}(t) \\
& \underline{\beta}_{3}(t)=\underline{\alpha}_{3}(t)-((T A-L C) N) v(t), \\
& \bar{\beta}_{1}(t)=\bar{\alpha}_{1}(t), \bar{\beta}_{2}(t)=\bar{\alpha}_{2}(t), \\
& \bar{\beta}_{3}(t)=\bar{\alpha}_{3}(t)+((T A-L C) N) v(t) .
\end{aligned}
$$

By using a similar argumentation, we get

$$
\begin{aligned}
& \dot{e}_{\chi}\left(t_{i+1}\right)=(R G-M C) \underline{e}_{\chi}\left(t_{i+1}^{-}\right)+\sum_{j=1}^{j=3} \underline{h}_{j}\left(t_{i+1}\right), \\
& \dot{\bar{e}}_{\chi}\left(t_{i+1}\right)=(R G-M C) \bar{e}_{\chi}\left(t_{i+1}^{-}\right)+\sum_{j=1}^{j=3} \bar{h}_{j}\left(t_{i+1}\right),
\end{aligned}
$$

with

$$
\begin{aligned}
\underline{h}_{1}\left(t_{i+1}\right) & =\left(M^{+} \bar{v}-M^{-} \underline{v}\right)-M v\left(t_{i+1}\right), \\
\underline{h}_{2}\left(t_{i+1}\right) & =R g\left(t_{i+1}\right)-\left(R^{+} \underline{g}-R^{-} \bar{g}\right), \\
\underline{h}_{3}\left(t_{i+1}\right) & =((R G-M C) J)^{+} \bar{v}-((R G-M C) J)^{-} \underline{v} \\
& -((R G-M C) J) v\left(t_{i+1}\right), \\
\bar{h}_{1}\left(t_{i+1}\right) & =M v\left(t_{i+1}\right)-\left(M^{+} \underline{v}-M^{-} \bar{v}\right), \\
\bar{h}_{2}\left(t_{i+1}\right) & =\left(R^{+} \bar{g}-R^{-} \underline{g}\right)-R g\left(t_{i+1}\right), \\
\bar{h}_{3}\left(t_{i+1}\right) & =-\left(((R G-M C) J)^{+} \underline{v}-((R G-M C) J)^{-} \bar{v}\right) \\
& +((R G-M C) N) v\left(t_{i+1}\right) .
\end{aligned}
$$

It can be inferred by applying Lemma 1 that the signals $\left\{\underline{\beta}_{i}(t), \bar{\beta}_{i}, 1 \leq i \leq 3\right\}$ are nonnegative for all $t \in\left[t_{i}, t_{i+1}\right)$ and the signals $\left\{\underline{h}_{j}\left(t_{i+1}\right), \bar{h}_{j}\left(t_{i+1}\right), 1 \leq j \leq 3\right\}$ are nonnegative $\forall i \in \mathbb{Z}_{\geq 0}$. Consequently, we deduce by using Lemma 2 and Lemma 3 that $\bar{e}_{\chi}(t) \geq 0, \underline{e}_{\chi}(t) \geq 0$ since $\underline{e}_{\chi}(0) \geq 0, \bar{e}_{\chi}(0) \geq 0$, the matrix $T A-L C$ is Metzler by using Assumption 1.ii and the matrix $R G-M C$ is nonnegative by using Assumption 1.iii (the systems (18) and (19) are cooperative). Accordingly, the order relation $\chi(t) \leq T x(t) \leq \bar{\chi}(t)$ is satisfied for all $t \in\left[t_{i}, t_{i+1}\right)$ and the order relation $\chi\left(t_{i+1}\right) \leq R x\left(t_{i+1}\right) \leq \bar{\chi}\left(t_{i+1}\right)$ holds.

Moreover, the inequalities $\underline{\chi}(t) \leq T x(t)$ for all $t \in$ $\left[t_{i}, t_{i+1}\right)$ and $\underline{\chi}\left(t_{i+1}\right) \leq R x\left(t_{i+1}\right)$ are equivalent to

$$
\begin{aligned}
& \underline{x}(t) \leq x(t)+N v(t)-\left(N^{+} \bar{v}-N^{-} \underline{v}\right), t \in\left[t_{i}, t_{i+1}\right), \\
& \underline{x}\left(t_{i+1}\right) \leq x\left(t_{i+1}\right)+J v\left(t_{i+1}\right)-\left(J^{+} \bar{v}-J^{-} \underline{v}\right) .
\end{aligned}
$$

By applying Lemma 1 , we deduce that $\underline{x}(t) \leq x(t)$ for all $t \in \mathbb{R}_{\geq 0}$. On the other hand, one can rewrite the inequality $T x(t) \leq \bar{\chi}(t)$ for all $t \in\left[t_{i}, t_{i+1}\right)$ and the inequality $R x\left(t_{i+1}\right) \leq \bar{\chi}\left(t_{i+1}\right)$ as follows

$$
\begin{aligned}
T x(t) \leq & \bar{x}(t)-N C x(t)-N v(t)-N^{-} \bar{v}+N^{+} \underline{v}, \\
R x\left(t_{i+1}\right) \leq & \bar{x}\left(t_{i+1}\right)-J C x\left(t_{i+1}\right)-J v\left(t_{i+1}\right)- \\
& J^{-} \bar{v}+J^{+} \underline{v},
\end{aligned}
$$

which is equivalent to

$$
\begin{aligned}
& x(t) \leq \bar{x}(t)+\left(N^{+} \underline{v}-N^{-} \bar{v}\right)-N v(t), t \in\left[t_{i}, t_{i+1}\right), \\
& x\left(t_{i+1}\right) \leq \bar{x}\left(t_{i+1}\right)+\left(J^{+} \underline{v}-J^{-} \bar{v}\right)-J v\left(t_{i+1}\right) .
\end{aligned}
$$

It can be inferred by using Lemma 1 that $x(t) \leq \bar{x}(t)$ for all $t \in \mathbb{R}_{\geq 0}$.

Furthermore, we conclude from Theorem 1 that the errors' systems (17) and (19) are asymptotically stable when Assumption 1.i holds. We deduce from Theorem 2 that $\underline{e}_{\eta}, \bar{e}_{\eta} \in \mathcal{L}_{\infty}^{n}$. From (11), we get

$$
\begin{aligned}
\underline{e}(t) & =\underline{e}_{\eta}(t)+N^{+} \bar{v}-N^{-} \underline{v}, \forall t \in\left[t_{i}, t_{i+1}\right), \\
\underline{e}\left(t_{i+1}\right) & =\underline{e}_{\eta}\left(t_{i+1}\right)+J^{+} \bar{v}-J^{-} \underline{v}, \\
\bar{e}(t) & =\bar{e}_{\eta}(t)+N^{-} \bar{v}-N^{+} \underline{v}, \forall t \in\left[t_{i}, t_{i+1}\right), \\
\bar{e}\left(t_{i+1}\right) & =\bar{e}_{\eta}\left(t_{i+1}\right)+J^{-} \bar{v}-J^{+} \underline{v},
\end{aligned}
$$

Hence, we get $\underline{e}, \bar{e} \in \mathcal{L}_{\infty}^{n}$.

\section{NUMERICAL SIMULATIONS}

In this section, we compare the results of this work to those of the existing literature. Consider an abstract model, where the matrices of the system (1) are defined as follows

$$
A=\left[\begin{array}{cc}
-1 & -3 \\
2 & -1
\end{array}\right] ; G=\left[\begin{array}{cc}
2 & -1 \\
1 & 0.6
\end{array}\right] ; C=\left[\begin{array}{cc}
1 & 0
\end{array}\right] .
$$

For the simulations, we select $v(t)=0.1 \sin \left(\frac{1}{2 \pi} t\right)$, and $w(t)=g(t)=\left[0.1 \sin \left(\frac{1}{2 \pi} t\right), 0.1 \sin \left(\frac{1}{2 \pi} t\right)\right]^{T}$. Impulses occur in the system (1) at $t=2.5$ and $t=5$. The unknown initial condition and its bounds are

$$
x(0)=\left[\begin{array}{l}
1.5 \\
1.5
\end{array}\right] ; \underline{x}(0)=\left[\begin{array}{l}
1.3 \\
1.3
\end{array}\right] ; \underline{x}(0)=\left[\begin{array}{l}
1.7 \\
1.7
\end{array}\right] .
$$

Notice that there exists no gain $L$ such that the matrix $(A-L C)$ is Metzler. Consequently, methodologies such as 


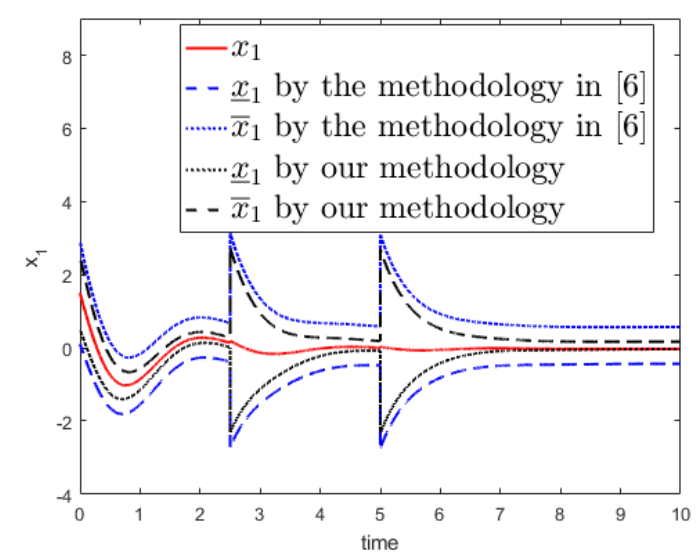

Fig. 1. Evolution of $x_{1}$ and the observed bounds $\underline{x}_{1}$ and $\bar{x}_{1}$

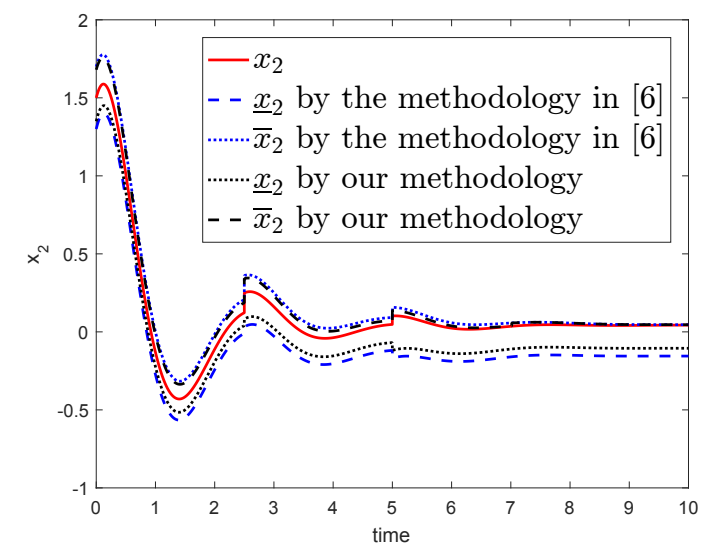

Fig. 2. Evolution of $x_{2}$ and the observed bounds $\underline{x}_{2}$ and $\bar{x}_{2}$

those that have been proposed in [7], [6, Theorem 3] cannot be applied to construct an interval estimator. Assumption 1.ii holds when we select $L=\left[\begin{array}{ll}5 & 0\end{array}\right]^{\mathrm{T}}$ and $T=$ $\left[\begin{array}{ll}0 & 0 \\ 2 & 1\end{array}\right]$ : the matrix $T A-L C=\left[\begin{array}{cc}-5 & 0 \\ 0 & -7\end{array}\right]$ is Metzler. Assumption 1.iii is satisfied with $M=\left[\begin{array}{ll}-0.5 & 0\end{array}\right]^{\mathrm{T}}$ and $R=\left[\begin{array}{cc}0 & 0 \\ -0.5 & 1\end{array}\right]:$ the matrix $R G-M C=\left[\begin{array}{cc}0.5 & 0 \\ 0 & 1.1\end{array}\right]$ is nonnegative. The matrix $N=\left[\begin{array}{ll}1 & -2\end{array}\right]^{\mathrm{T}}$ and the matrix $J=\left[\begin{array}{ll}1 & 0.5\end{array}\right]^{\mathrm{T}}$. By using Matlab YALMIP toolbox [16] with SeDuMi to solve the LMIs, one can find that Assumption 1.i holds for $T_{\min }=0.22$.

We compare on Fig. 1 and Fig. 2 the estimates given by the interval observer (11) with those given by using the coordinate transformation methodology proposed in $[6$, Theorem 4]. We remark that the interval observer (11) provides the most accurate interval estimates.

\section{CONCLUSiON}

We have considered the problem of interval observer design for uncertain linear impulsive systems. The model uncertainties are assumed to be unknown with given bounds. We have proposed a new structure to circumvent the issues of transformation of coordinates by designing an interval observer with more degrees of freedom. We have illustrated the performance of the proposed methodology through numerical simulations. Future research can extend the methodology to nonlinear systems.

\section{REFERENCES}

[1] G. Besançon, Nonlinear observers and applications. Springer, 2007, vol. 363.

[2] B. D. Anderson and J. B. Moore, Optimal filtering. Dover, 2012.

[3] F. Mazenc and O. Bernard, "Asymptotically stable interval observers for planar systems with complex poles," IEEE Transactions on Automatic Control, vol. 55, no. 2, pp. 523-527, 2010.

[4] K. H. Degue, D. Efimov, and J. Le Ny, "Interval observer-based feedback control for rehabilitation in tremor," in 2020 European Control Conference (ECC), 2020, pp. 1585-1590.

[5] K. H. Degue, D. Efimov, and J.-P. Richard, "Interval observers for linear impulsive systems," in Proc. of 10th IFAC Symposium on Nonlinear Control Systems (NOLCOS 2016), Monterey, California, USA, August 2016.

[6] — - "Stabilization of linear impulsive systems under dwell-time constraints: Interval observer-based framework," European Journal of Control, vol. 42, pp. 1-14, Jul. 2018.

[7] C. Briat and M. Khammash, "Simple interval observers for linear impulsive systems with applications to sampled-data and switched systems," IFAC-PapersOnLine, vol. 50, no. 1, pp. 5079-5084, July 2017.

[8] Z. Wang, C.-C. Lim, and Y. Shen, "Interval observer design for uncertain discrete-time linear systems," Systems \& Control Letters, vol. 116, pp. 41 - 46, Jun. 2018.

[9] W. Tang, Z. Wang, and Y. Shen, "Interval estimation for discretetime linear systems: A two-step method," Systems \& Control Letters, vol. 123, pp. 69 - 74, Jan. 2019.

[10] R. Goebel, R. G. Sanfelice, and A. R. Teel, Hybrid Dynamical Systems: Modeling, Stability, and Robustness. Princeton University Press, 2012.

[11] D. Efimov, L. Fridman, T. Raïssi, A. Zolghadri, and R. Seydou, "Interval estimation for LPV systems applying high order sliding mode techniques," Automatica, vol. 48, pp. 2365-2371, 2012.

[12] L. Farina and S. Rinaldi, Positive Linear Systems: Theory and Applications. New York: Wiley, 2000.

[13] C. Briat and A. Seuret, "Convex dwell-time characterizations for uncertain linear impulsive systems," IEEE Transactions on Automatic Control, vol. 57, no. 12, pp. 3241-3246, 2012.

[14] J. Hespanha, D. Liberzon, and A. R. Teel, "Lyapunov conditions for input-to-state stability of impulsive systems," Automatica, vol. 44, no. 11, pp. 2735-2744, 2008.

[15] Z. Wang, Y. Shen, X. Zhang, and Q. Wang, "Observer design for discrete-time descriptor systems: An LMI approach," Systems \& Control Letters, vol. 61, no. 6, pp. 683-687, Jun. 2012.

[16] J. Löfberg, "Yalmip : A toolbox for modeling and optimization in matlab," Automatic Control Laboratory,ETHZ, 2004. 